\title{
Influência da camada de microrrevestimento asfáltico a frio no aumento da vida útil dos pavimentos de segmentos monitorados em Araranguá
}

\author{
Aline Selau Santos ${ }^{1}$ \\ Glicério Trichês ${ }^{2}$
}

Resumo: O presente artigo avalia o acréscimo de vida útil de pavimentos asfálticos utilizandose da técnica de microrrevestimento asfáltico a frio - MRAF para a manutenção preventiva em rodovias de elevado volume de tráfego. Para o desenvolvimento do trabalho, foram utilizados dados das condições funcionais e estruturais de segmentos monitorados inseridos na Rodovia BR-101/SC, no município de Araranguá. Em todos os segmentos, empregou-se no revestimento uma mistura asfáltica com CAP 50/70, convencional, dosada pela metodologia Superpave, variando-se a espessura entre 15,0 e 18,0 cm. As misturas asfálticas foram caracterizadas quanto à deformação permanente, módulo complexo e fadiga. Com os dados dos ensaios de laboratório e dos valores de deflexões obtidos por retroanálise das estruturas no ano de abertura ao tráfego, foram realizadas simulações numéricas com o programa ViscoRoute, levando-se em conta a temperatura da região onde está inserida a rodovia. A partir da deformação máxima específica de tração obtida na face inferior da camada de revestimento, foi possível estimar a vida útil de cada segmento em termos de número de solicitações do eixo padrão de 8,2tf (USACE). A rodovia foi aberta ao tráfego em março de 2014. Desde então, foram realizados sete levantamentos das condições funcionais e estruturais, dando-se ênfase à evolução da porcentagem da área trincada, Em três dos segmentos (pista sentido sul) foi aplicada uma camada de microrrevestimento como estratégia de manutenção preventiva da rodovia, pois já se observava, naquele momento, o aparecimento de trincas no revestimento. Nos três segmentos localizados no sentido norte, não houve aplicação do microrrevestimento. O comparativo entre a vida útil estimada pelo ViscoRoute, o tráfego atuante e a evolução da porcentagem da área trincada, possibilitou que fossem estabelecidos fatores de calibração entre laboratório e campo, bem como, a avaliação da influência da aplicação do microrrevestimento na ampliação da vida útil do revestimento. Considerando-se a porcentagem de área trincada como variável de referência, os resultados permitiram concluir, que nos segmentos em que o microrrevestimento foi aplicado já no início do aparecimento das primeiras trincas, o aumento da vida útil chega a 98\% da vida útil esperada para os segmentos que não receberam esta solução.

Palavra-chave: Pavimentação. Microrrevestimento. Vida Útil de pavimentos

\section{Influence of the cold asphalt micro-coating layer in increasing the service life of the floors of monitored segments in Araranguá}

\footnotetext{
${ }^{1}$ Mestre em Engenharia Civil. aline.selau@gmail.com

${ }^{2}$ Doutor em Engenharia Civil, Professor UNESC, glicerio.triches@unesc.net
} 
Abstract: This Present article evaluates the adding of asphaltic pavements life span, using the cold asphaltic micro- coverage technique - AMRT (MRAF in Portuguese) for the preventive maintenance of high-volume traffic highways. For this article's development we used functional and structural data from segments inserted and monitored at Highway BR-101/SC in the city of Araranguá. In all segments an asphaltic mixture of CAP 50/70 was employed, conventional dosed by the methodology Super Pave, varying thickness between 15,0 and 18,0 $\mathrm{cm}$. The asphaltic mixtures were categorized according to their permanent defect/deformation complex module and fatigue. With laboratory data from the experiments and from the values of deflections obtained by retro analysis from the structures at the year of traffic opening, numerical simulations with the Viscoroute program were realized. Taking into consideration the regions temperature where the road was. Strategic at the highest specific deformation from obtained traction at the lower layer of the pavement. It was possible then to calculate/estimate the life span of each segment in terms of numbers of demands of standard axes 8,2tf (USACE). The highway was open to public on March 2014. Since then 7 appraisals of functional and structural conditions were conducted. Giving emphasis at the percentage evolution of the cracked parts. Among three of the segments (south road) it was applied a layer of micro coverage as a means of strategy for preventive maintenance at the highway for there could be already observed at that time signs of cracks at the pavement coverage. At the three segments found on the north road micro pavement coverage wasn't applied. The comparative between the life span estimated by the ViscoRoute. The acting traffic and the evolution of percentages of cracked areas as a variable of reference, the results allowed for closure, that at the segments in which the micro pavement coverage was applied since the beginning at the appearances of the first signs of cracking, the increase of the life span expected can go up to $98 \%$ compared to the ones that didn't receive this mixture.

Key words: Pavement, Micro Coverage. Pavement's life span.

\section{Introdução}

A elevada incidência de tráfego de veículos pesados e o baixo investimento destinado a manutenção da malha viária brasileira, aliados a problemas como deficiências no método de dimensionamento, falta de caracterização dos materiais empregados, falhas na execução e a falta de controle sistemático do excesso de carga dos veículos, acarreta no aparecimento precoce de muitos defeitos nos pavimentos, principalmente, aqueles relacionados com o trincamento e afundamento na trilha de roda.

O comportamento dos pavimentos frente às solicitações a que são impostos é de grande complexidade devido à junção de vários tipos de materiais em uma mesma estrutura, além da influência das variações climáticas e ambientais. Este comportamento passa a ser melhor 


\section{Revista Técnico-Cientifica de Engenharia Civil CIVILTEC}

entendido a partir da realização de ensaios de laboratório que buscam simular as condições mais próximas daquelas encontradas em campo e da análise sistematizada do desempenho em serviço (monitoramento).

A partir da caracterização do comportamento dos materiais em laboratório e campo e a aplicação de softwares que possibilitam calcular as tensões, deformações e deslocamentos do sistema de camadas em relação ao carregamento aplicado é possível prever o comportamento da estrutura e estimar a vida útil que será alcançada.

Por sua vez, os dados de campo obtidos no monitoramento oferecem a possibilidade de comparar o desempenho real dos pavimentos com o desempenho estimado através de análises mecanicistas, sendo possível encontrar funções de transferência (fatores de calibração) entre campo e laboratório. Com isso, pavimentos com estruturas e materiais semelhantes, sujeitas às mesmas configurações de tráfego, podem ter seu desempenho correlacionados.

Para o desenvolvimento do presente trabalho, utilizou-se os resultados do monitoramento dos segmentos localizados na Rodovia BR-101/SC. A construção e monitoramento dos segmentos foi realizada pela Universidade Federal de Santa Catarina com apoio do DNIT, através de um projeto de pesquisa financiado pela Rede Temática de Tecnologia do Asfalto, cujo objetivo foi contribuir para o desenvolvimento do novo método brasileiro de dimensionamento de pavimentos flexíveis, o MEDINA.

Foram construídos 5 segmentos, sendo dois deles localizados na pista sul (sentido nortesul) e três na pista norte (sentido sul-norte). Os segmentos foram liberados ao tráfego em março de 2014. Com base nas informações extraídas dos trabalhos de Trichês (2014), Santos (2016), Luz (2017) e Lopes (2019) foi possível obter as características de cada segmento com relação aos tipos de materiais utilizados, espessura das camadas e desempenho da mistura asfáltica decorrentes dos ensaios de laboratório e das avaliações de campo.

O diagrama unifilar da Figura 1 mostra a localização dos 5 segmentos. Especificamente para esta pesquisa foi escolhido um sexto segmento, contíguo ao trecho monitorado, que serviu como segmento de referência por representar a estrutura executada em todo o trecho rodoviário em que os segmentos estão inseridos.

De forma a poder preservar as características estruturais e funcionais da rodovia, o DNIT, responsável pela manutenção e conservação do local optou por realizar a reabilitação do pavimento através da aplicação de microrrevestimento asfáltico a frio - MRAF, o que foi feito 


\section{Revista Técnico-Cientifica de Engenharia Civil CIVILTEC}

após um ano de abertura ao tráfego. Para que o monitoramento pudesse continuar, foi aplicado MRAF apenas nos segmentos da pista sul, ficando os segmentos da pista norte sem a aplicação de micro.

Este trabalho apresenta o desempenho quanto à vida útil dos segmentos que receberam a camada de MRAF em relação aos segmentos em que não foi aplicada essa estratégia de manutenção preventiva. Além disso, mostra um comparativo entre os gastos da administração pública com a solução utilizada e os gastos com a restauração convencional.

Figura 1: Esquema de localização dos segmentos monitorados

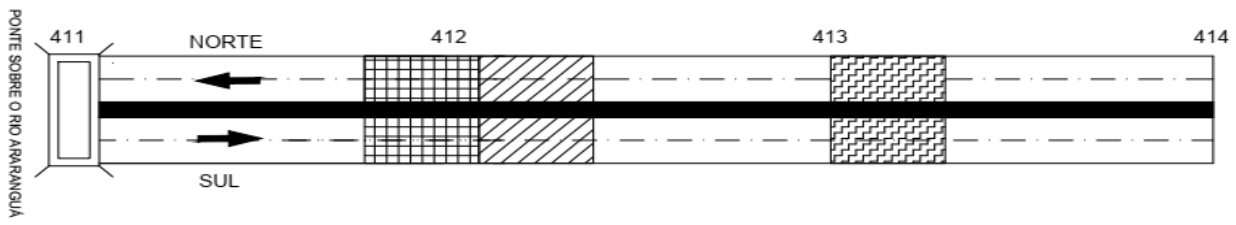

\section{CONVENÇŌES:}

SEGMENTOS 1 (SUL) E 5 (NORTE) - km 411,79 - km 412,09

DIJ SEGMENTOS 2 (SUL) E 3 (NORTE) - km 412,09 - km 412,39

FE] SEGMENTOS 4 (NORTE) E 6 (SUL) - km 413,0 - km 413,3

Fonte: dados da pesquisa

A Tabela 1 mostra o tipo e a espessura de material utilizado em cada camada do pavimento dos segmentos.

Tabela 1: Espessura das camadas do pavimento e tipos de materiais empregados

\begin{tabular}{lcccccc}
\hline \multicolumn{1}{c}{ SEGMENTOS } & 01 & 02 & 03 & 04 & 05 & 06 \\
\hline Revestimento - Mistura asfáltica CAP 50/70) & 16,5 & 18,0 & 11,4 & 15,2 & 16,3 & 15,0 \\
Revestimento - Mistura asfáltica (ECOFLEX & & & & & & - \\
B) & - & - & 5,6 & - & - & 18 \\
Base - Brita Graduada & 18 & 18 & 18 & 18 & 18 & 20 \\
Sub- base - Macadame Seco & 20 & 20 & 20 & 20 & 20 & $\geq 60$ \\
Subleito - Areia & $\geq 60$ & $\geq 60$ & $\geq 60$ & $\geq 60$ & $\geq 60$ & $\geq 60$ \\
\hline
\end{tabular}

Fonte: dados da pesquisa.

Ressalta-se que o segmento 3 não será objeto deste estudo, pois o revestimento é diferenciado em relação aos outros segmentos, e, portanto, não poderia estar numa mesma análise.

\section{Materiais e método}

A mistura asfáltica utilizada nos segmentos empregou agregados de origem basáltica. A definição da composição granulométrica foi feita pelo Centro de Estudos e Desenvolvimento 


\section{Revista Técnico-Cientifica de Engenharia Civil CIVILTEC}

Leopoldo Américo Miguez de Mello - CENPES/Petrobrás visando obter uma mistura com bom desempenho quanto à deformação permanente.

A granulometria escolhida apresentou comportamento graúdo, se enquadra nos limites da faixa B do DNIT (2010), com tamanho máximo do agregado de 19,1 mm. Para a composição da mistura foram utilizados $43 \%$ de brita $3 / 4 "$ (19,1 mm), 18,5\% de brita 3/8" $(9,1 \mathrm{~mm}), 37 \%$ de pó de pedra $(4,75 \mathrm{~mm})$ e 1,5\% de fíler (cal hidratada $\mathrm{CH}-01)$.

O ligante asfáltico utilizado na camada de rolamento foi o CAP-50/70. A dosagem da mistura foi realizada pelo Cenpes/Petrobrás utilizando a metodologia Superpave. A Tabela 2 mostra os parâmetros volumétricos da mistura asfáltica.

Tabela 2: Parâmetros Volumétricos da Mistura Asfáltica

\begin{tabular}{ccccccc}
$\begin{array}{c}\text { Teor de ligante } \\
(\%)\end{array}$ & $\begin{array}{c}\text { Vazios } \\
(\%)\end{array}$ & $\begin{array}{c}\text { VAM } \\
(\%)\end{array}$ & $\begin{array}{c}\text { RBV } \\
(\%)\end{array}$ & $\begin{array}{c}\text { RFB } \\
(\%)\end{array}$ & $\begin{array}{l}\mathrm{Gmm}\left(\mathrm{g} / \mathrm{cm}^{3}\right) \\
\left(\mathrm{g} / \mathrm{cm}^{3}\right)\end{array}$ \\
\hline 4,15 & 4 & 13,2 & 69,7 & 2,3 & 2,705 & 2,597 \\
\hline
\end{tabular}

Fonte: Cenpes (2010).

A mistura asfáltica foi caracterizada com relação ao desempenho à deformação permanente através do ensaio de cargas rolantes em laboratório, preconizado através da norma francesa AFNOR NF P 98-253 - Essas Relatifs Aux Chaussées, Déformation Permanente des Mélanges Hydrocarnoné.

O equipamento utilizado consiste em um simulador de tráfego dotado de um eixo simples de roda simples que percorre a superfície de duas placas de concreto asfáltico, simultaneamente, a uma frequência de $1 \mathrm{~Hz}$. O carregamento aplicado é de $5 \mathrm{kN}$ e a pressão de inflação dos pneus é de $0,6 \mathrm{MPa}$. $\mathrm{O}$ ensaio é conduzido a uma temperatura de $60^{\circ} \mathrm{C}$ e as placas são submetidas a 30000 ciclos. A Tabela 3 mostra o resultado obtido para a mistura asfáltica empregada nos segmentos. As placas ensaiadas foram extraídas em campo e moldadas em laboratório.

Tabela 3: Resultado do ensaio de deformação permanente da mistura asfáltica empregada nos segmentos

\begin{tabular}{cccccc}
\hline Mistura & Identificação & Placa & $\begin{array}{c}\mathrm{Gmb} \\
\left(\mathrm{g} / \mathrm{cm}^{3}\right)\end{array}$ & $\mathrm{Vv}(\%)$ & $\begin{array}{c}\text { Deformação } \\
\text { Permanente média }(\%)\end{array}$ \\
\hline \multirow{2}{*}{$\begin{array}{c}\text { Mistura } \\
\text { asfáltica }\end{array}$} & Placas moldadas em & 1 & 2,52 & 6,13 & 9,5 \\
convencional & Plaboratório & 2 & 2,564 & 4,5 & \\
& campo & Estaca 0+680 BE & 2,501 & 7,56 & 6,4 \\
\hline
\end{tabular}

Fonte: Trichês (2014). 


\section{Revista Técnico-Cientifica de Engenharia Civil CIVILTEC}

O limite para este ensaio é de 5\% para tráfegos pesados e 10\% para tráfegos de médio a pesado, segundo a especificação francesa. Normalmente, valores abaixo de 5\% somente são alcançados com o uso de CAPs modificados. Portanto, a partir dos materiais disponíveis para a dosagem, o valor de 6,4\% para mistura com ligante convencional pode ser considerado satisfatório.

Para caracterização do comportamento reológico das misturas de concreto asfáltico produzidas foi realizado o ensaio de módulo complexo a diversas frequências e temperaturas de teste. O ensaio seguiu as diretrizes da norma europeia EN 12697-26 - Bituminous Mixtures, Test Methods for Hot Mix Asphalt - Part 26: Stiffness e foi conduzido sob carregamento sinusoidal contínuo e deformação controlada $(50 \mu \mathrm{m} / \mathrm{m})$ empregando-se o equipamento aparato de 4 pontos.

A partir dos resultados do ensaio e com auxílio do software ViscAnalyse, determinouse os parâmetros reológicos do modelo de Huet-Sayegh de cada mistura, como mostra a Tabela 4. Estes resultados foram utilizados na análise tensão-deformação das estruturas para a estimativa da vida útil empregando-se o software VicoRoute.

\begin{tabular}{c|c} 
Tabela 4: Parâmetros reológicos do modelo de Huet-Sayegh \\
\hline Parâmetros & Campo \\
\hline Eœ (MPa) & 29238,2 \\
$v$ & 0,30 \\
E0 (MPa) & 76,5434 \\
k & 0,24856 \\
h & 0,68540 \\
$\delta$ & 2,70810 \\
A0 & 5,89107 \\
A1 & $-0,436450$ \\
A2 & 0,002040 \\
\hline
\end{tabular}

Fonte: Trichês (2014).

A determinação da resistência à fadiga da mistura asfáltica seguiu diretrizes da norma EN 12697-24 - Bituminous Mixtures, Test Methods for Hot Mix Asphalt - Part 24: Resistance to Fatigue. O ensaio foi conduzido sob carregamento sinusoidal contínuo e deformação controlada $(50 \mu \mathrm{m} / \mathrm{m})$ empregando-se o equipamento aparato de 4 pontos. O critério de ruptura foi a redução de $50 \%$ do módulo complexo inicial, com módulo inicial determinado no centésimo ciclo. A temperatura do ensaio definida com base no comportamento reológico das misturas de concreto asfáltico, segundo o plano complexo (Cole-Cole) em termos de maior 


\section{Revista Técnico-Cientifica de Engenharia Civil CIVILTEC}

dissipação de energia e a frequência de teste de $10 \mathrm{~Hz}$. Os ensaios foram realizados com placas moldadas em laboratório e ensaiados a temperatura de $15^{\circ} \mathrm{C}($ Melo,2014) e com placas extraídas em campo, ensaiadas a temperatura de $15^{\circ} \mathrm{C}$ e $20^{\circ} \mathrm{C}$ (Trichês, 2014).

O modelo que apresentou relação mais próxima com o que ocorre em campo (menor fator laboratório/campo) foi o modelo de Trichês (2014) obtido a partir de corpos de prova extraídos de campo e ensaiados a $15^{\circ} \mathrm{C}$, na qual se obteve a seguinte equação 1:

$$
\mathrm{N}=9,72 \times 10^{14} \varepsilon \mathrm{t}^{-4,742}
$$

Onde: N: número para redução de 50\% do módulo complexo inicial

et: deformação de tração inicial (obtida nos 100 ciclos de aplicação do carregamento).

Os agregados utilizados na produção do MRAF são de origem basáltica. A caracterização dos agregados envolveu ensaios de abrasão Los Angeles (DNER-ME-35/98), durabilidade (DNER-ME 089/94), equivalente de areia (DNER 054/97) e azul de metileno (NBR 14949). Os resultados atenderam à especificação brasileira DNIT 035/2005 com relação à seleção dos agregados para o MRAF.

A granulometria empregada foi a faixa II da Norma DNIT 035/2005 que é recomendada para rodovias de tráfego intenso e pesado e de alta amplitude térmica, como é o caso da rodovia BR-101/SC. A composição da mistura foi de $75 \%$ pó de pedra, $24 \%$ de pedrisco e $1 \%$ de fíler. O fíler mineral utilizado no traço foi a cal hidratada $\mathrm{CH}-1$.

A emulsão asfáltica utilizada foi a Emulex RC 1C-E, que é uma emulsão catiônica especial de ruptura controlada modificada por polímero elastomérico. $\mathrm{O}$ teor foi de $9,4 \%$. Esse teor foi calculado considerando-se $62 \%$ de resíduo asfáltico teórico mínimo.

Foi utilizada a proporção de $0,5 \%$ em peso dos agregados de aditivo controlador de ruptura da emulsão asfáltica. O produto utilizado foi à base de tensoativos em solução iônica, denominado Adiflex Arc.

O teor de água da mistura foi definido no projeto de dosagem como sendo 9,3\%.

Para a verificação da compatibilidade dos materiais na mistura foram realizados os ensaios mostrados a Tabela 5 (Ceratti, et al, 2015). 


\section{Revista Técnico-Cientifica de Engenharia Civil CIVILTEC}

Tabela 5: Ensaios da mistura de MRAF

\begin{tabular}{|c|c|c|c|}
\hline Ensaio & Norma & Resultado & Especificação \\
\hline $\begin{array}{l}\text { Determinação da coesão e características de cura } \\
\text { pelo coesímetro (MCT). }\end{array}$ & NBR 14798 & $25 \mathrm{~kg} . \mathrm{cm}$ & $20 \mathrm{~kg} . \mathrm{cm}$ \\
\hline Determinação da adesividade de misturas (WST). & NBR 14757 & satisfatório & satisfatório \\
\hline $\begin{array}{l}\text { Determinação da perda por abrasão úmida } \\
\text { (WTAT). }\end{array}$ & $\begin{array}{l}\text { NBR } 14746 / \\
\text { ISSA A-143 }\end{array}$ & $295,2 \mathrm{~g} / \mathrm{m}^{2}$ & satisfatório/ $<538 \mathrm{~g} / \mathrm{m}^{2}$ \\
\hline $\begin{array}{l}\text { Determinação do excesso de asfalto e adesão de } \\
\text { areia pela máquina (LWT) }\end{array}$ & NBR 14841 & $430,7 \mathrm{~g} / \mathrm{m}^{2}$ & $538 \mathrm{~g} / \mathrm{m}^{2}$ \\
\hline
\end{tabular}

Fonte: dados da pesquisa.

As características funcionais dos pavimentos foram analisadas entre o ano de abertura, (2014) e o ano de 2019, ou seja, cinco anos de monitoramento. O tráfego foi atualizado a partir dos dados do Plano Nacional de Contagem de Tráfego. O número $\mathrm{N}$ acumulado para o período de vida útil de projeto foi de $2,35 \times 10^{7}$ determinado pelo método da AASTHO (American Association of State Highway Transportation Officials) e $8,02 \times 10^{7}$ pela metodologia do USACE (Corpo de Engenheiros do Exército Americano).

O monitoramento seguiu a cronologia mostrada na Tabela 6. É importante salientar que os segmentos 01, 02 e 06 são os segmentos que receberam microrrevestimento após 1 ano de serviço, desta forma, ficaram de fora dos demais levantamentos.

Tabela 6: Cronologia dos levantamentos

\begin{tabular}{|c|c|c|c|c|}
\hline Ano & Meses & $\begin{array}{l}\text { Segmentos } \\
\text { analisados }\end{array}$ & Ensaios & $\begin{array}{l}\mathrm{N}_{\text {atuante }} \\
\text { (USACE) }\end{array}$ \\
\hline $2014^{(1)}$ & 0 & 01,02 e 03 & $\begin{array}{l}\text { IRI, ATR, deflexão (FWD), macro e } \\
\text { microtextura e defeitos }\end{array}$ & 0 \\
\hline $2015^{(2)}$ & 12 & 03,04 e 05 & ATR, macro e microtextura e defeitos & $9,07 \times 10^{6}$ \\
\hline $2016^{(2)}$ & 26 & 03,04 e 05 & $\begin{array}{l}\text { IRI, ATR, deflexão (FWD), macro e } \\
\text { microtextura e defeitos }\end{array}$ & $1,78 \times 10^{7}$ \\
\hline $2017^{(2)}$ & 40 & 03,04 e 05 & ATR, macro e microtextura e defeitos & $2,65 \times 10^{7}$ \\
\hline $2017^{(3)}$ & 46 & 03,04 e 05 & IRI, ATR, deflexão (FWD) & $2,98 \times 10^{7}$ \\
\hline $2018^{(3)}$ & 57 & 03,04 e 05 & ATR, macro e microtextura e defeitos & $3,71 \times 10^{7}$ \\
\hline $2019^{(1)}$ & 70 & 01,02 e 06 & ATR e defeitos & $4,55 \times 10^{7}$ \\
\hline
\end{tabular}

Os segmentos foram analisados em dezembro de 2019, portanto 70 meses após a abertura ao tráfego. O método utilizado na avaliação foi o preconizado na Norma DNIT 007/2003 - PRO. Na ocasião foram levantados os afundamentos de trilha de rodas (ATR) e os defeitos de superfície. A área trincada foi previamente delimitada e medida. A área trincada foi separada nos tipos FC-1, FC-2 e FC-3. 


\section{Revista Técnico-Cientifica de Engenharia Civil CIVILTEC}

Os levantamentos ocorreram apenas nos segmentos 01, 02 e 06, que são os segmentos que possuem a camada de microrrevestimento asfáltico a frio.

\section{Resultados e Discussões}

O levantamento dos defeitos ocorreu apenas na faixa direita (faixa mais solicitada) de cada segmento. Houve a ocorrência apenas de trincas FC-1 e FC-2. A partir da medição da área trincada, determinaram-se as porcentagens de área trincada em relação à área total da faixa direita, com 3,6 metros de largura, e em relação somente a área da trilha de roda, ou seja, 2,4 metros de largura, conforme mostra a Tabela 7.

Tabela 7: Porcentagem de área trincada em relação a faixa direita e em relação a trilha de roda

\begin{tabular}{cccc}
\hline Segmento & $\begin{array}{c}\text { Espessura dos } \\
\text { revestimentos }(\mathrm{cm})\end{array}$ & $\begin{array}{c}\% \text { área trincada } \\
\text { em relação a faixa direita }\end{array}$ & $\begin{array}{c}\% \text { área trincada } \\
\text { em relação a trilha de roda }\end{array}$ \\
\hline 01 & $16,5+1,5$ de micro & 0,5 & 0,7 \\
02 & $18,0+1,5$ de micro & 0,4 & 0,6 \\
06 & $15,0+1,5$ de micro & 4,0 & 5,9 \\
\hline
\end{tabular}

Fonte: dados da pesquisa.

A Tabela 8 mostra a evolução da porcentagem da área trincada na faixa de rolamento da direita obtida por Lopes (2019) nos segmentos 04 e 05. Na Figura 2, a linha em vermelho representa a recomendação de Brasil (2006) para a necessidade de execução de remendos superficiais.

Tabela 8: Evolução da área trincada nos segmentos que não receberam microrrevestimento

\begin{tabular}{ccccccc}
\hline Ano & $\begin{array}{c}\text { Meses de abertura } \\
\text { ao tráfego }\end{array}$ & $\mathrm{N}$ atuante & $\mathrm{N}$ projeto & \multicolumn{2}{c}{$\mathrm{N}$ atuante/N } & \multicolumn{2}{c}{ Área trincada (\%) } \\
projeto $(\%)$ & S04 & S05 \\
\hline $2015^{(1)}$ & 13 & $9,07 \mathrm{E}+06$ & & 0,11 & 7,14 & 2,75 \\
$2016^{(1)}$ & 27 & $1,78 \mathrm{E}+07$ & $8,02 \mathrm{E}+07$ & 0,22 & 24,17 & 9,87 \\
$2017^{(1)}$ & 41 & $2,65 \mathrm{E}+07$ & & 0,33 & 28,6 & 13,01 \\
$2018^{(2)}$ & 57 & $3,70 \mathrm{E}+07$ & & 0,46 & 38,6 & 24,95 \\
\hline
\end{tabular}

Fonte: Adaptado de Luz (2017) ${ }^{(1)}$ e Lopes (2019) ${ }^{(2)}$

Considerando-se a hipótese de que os segmentos 01, 02 e 06 possam vir a ter uma evolução da porcentagem da área trincada semelhante àquela obtida nos segmentos 04 e 05 mostrada na Figura 2, fez-se uma estimativa do tráfego para que sejam atingidas naqueles segmentos as mesmas porcentagens de áreas trincadas. Ressalta-se que na época em que foram feitos os levantamentos nos segmentos que receberam a camada de microrrevestimento, o tráfego atuante era de 4,59x107 (USACE). 


\section{Revisto Térico-Cientifica de Engenharia Civil CIVILTEC}

Figura 2: Gráfico da evolução da área trincada nos segmentos que não receberam microrrevestiment

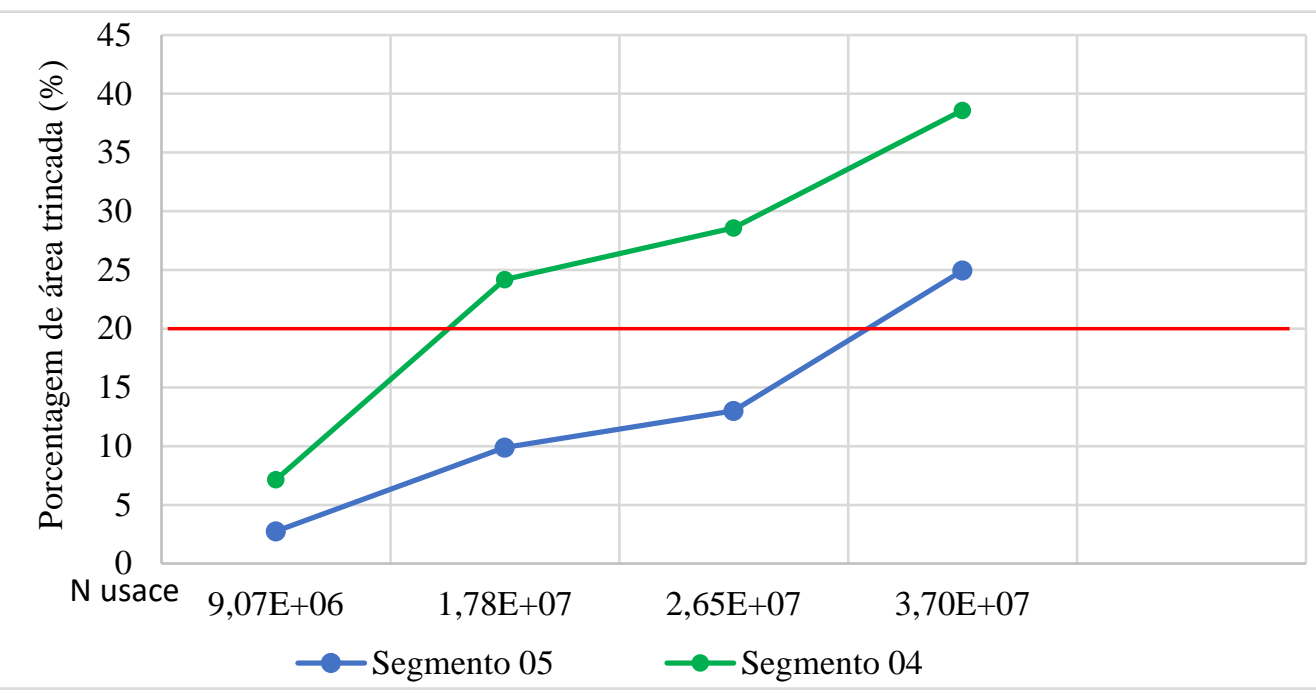

Assim, o tráfego para que ocorra $10 \%$ da área trincada no segmento 06 (com 15,0cm de espessura de revestimento e com micrrorrevestimento) corresponde ao tráfego na data do levantamento $(4,59 \mathrm{E}+07)$ mais o tráfego para se atingir $10 \%$ de área trincada. Admitindo-se que a evolução do trincamento no segmento 6 seja semelhando à do segmento $4(15,0 \mathrm{~cm}$ de revestimento e sem camada de micrrorevestimento) o tráfego para se atingir o grau de trincamento de $10 \%$ pode ser estimado a partir da Figura 2.

A Tabela 9 apresenta a estimativa do acréscimo tráfego para se ter a mesma porcentagem de área trincada nos segmentos que receberam o microrrevestimento a partir destas considerações. Os valores para o segmento $02(18,0 \mathrm{~cm}$ de revestimento) foram estimados a partir da modelação dos resultados para revestimento de 15,0 e $16,5 \mathrm{~cm}$ e considerando-se crescimento linear da porcentagem da área trincada.

Como pode ser observado na Tabela 9, a aplicação da camada de microrrevestimento pode aumentar a vida útil do pavimento de $52 \%$ a $98 \%$, dependendo da espessura do revestimento e da porcentagem da área trincada no momento de aplicação desta alternativa de manutenção preventiva. Ao se levar em conta que a espessura da camada de microrrevestimento propicia uma pequena redução na deformação de tração no revestimento e que esta camada retarda a penetração da água na estrutura do pavimento, é possível que a velocidade de crescimento da porcentagem da área trincada nos segmentos que receberam a camada de microrrevestimento venha até ser menor que aquela observada nos segmentos em a camada não 


\section{Revista Técnico-Cientifica de Engenharia Civil CIVILTEC}

foi aplicada. Portanto, é de se esperar que a porcentagem de acréscimo do tráfego seja até levemente maior do que aquela apresentada na Tabela 9.

Tabela 9: Estimativa do acréscimo tráfego para se ter a mesma porcentagem de área trincada nos segmentos que receberam o microrrevestimento

\begin{tabular}{|c|c|c|c|c|c|c|c|}
\hline \multirow[b]{2}{*}{ Segmento } & \multirow{2}{*}{$\begin{array}{l}\text { Porcentagem } \\
\text { de área } \\
\text { trincada para } \\
\text { tráfego atual }\end{array}$} & \multicolumn{3}{|c|}{ Para $10 \%$ de área trincada } & \multicolumn{3}{|c|}{ Para $20 \%$ de área trincada } \\
\hline & & $\begin{array}{l}\text { Acréscimo } \\
\text { do tráfego }\end{array}$ & $\begin{array}{l}\text { Tráfego } \\
\text { estimado }\end{array}$ & $\begin{array}{c}\% \text { de } \\
\text { acréscimo } \\
\text { do tráfego }\end{array}$ & $\begin{array}{l}\text { Acréscimo } \\
\text { do tráfego }\end{array}$ & $\begin{array}{l}\text { Tráfego } \\
\text { estimado }\end{array}$ & $\begin{array}{c}\% \text { de } \\
\text { acréscimo } \\
\text { do } \\
\text { tráfego }\end{array}$ \\
\hline 01 & $\begin{array}{l}0,5 \% \\
0,4 \%\end{array}$ & $2,4 \mathrm{E}+07$ & $7,0 \mathrm{E}+07$ & $\begin{array}{l}52,3 \\
87,0 *\end{array}$ & $3,8 \mathrm{E}+07$ & $8,4 \mathrm{E}+07$ & 83,0 \\
\hline 02 & & $4,0 \mathrm{E}+07 *$ & $8,6 \mathrm{E}+07 *$ & & $3,7 \mathrm{E}+07 *$ & $9,8 \mathrm{E}+07 *$ & $98,0 *$ \\
\hline 06 & $4,0 \%$ & $0,8 \mathrm{E}+07$ & $5,4 \mathrm{E}+07$ & 17,4 & $2,4 \mathrm{E}+07$ & $7,0 \mathrm{E}+07$ & 52,3 \\
\hline
\end{tabular}

O Afundamento da Trilha de Roda (ATR) foi medida somente na trilha de roda externa da pista da direita. A Tabela 10 apresenta o afundamento da trilha de roda média (ATR) e o desvio padrão $(\mathrm{Sd})$ em cada segmento.

Como esperado, o segmento 06 (revestimento de 15,0 cm) apresenta maiores valores de afundamento em decorrência, também, do trincamento, pois com que a infiltração de água na estrutura, aumenta a deformabilidade dos materiais, o bombeamento de finos e, em consequência, o aumento do afundamento na trilha de roda.

Tabela 10: Média e desvio padrão das medidas de ATR

\begin{tabular}{ccc}
\hline Segmento & ATR $(\mathrm{mm})$ & $\mathrm{Sd}(\mathrm{mm})$ \\
\hline 01 & 4,69 & 1,40 \\
02 & 4,63 & 1,02 \\
06 & 8,19 & 1,76 \\
\hline
\end{tabular}

Fonte: dados da pesquisa

Comparando-se os valores obtidos nesta pesquisa para os segmentos 01, 02 e 06 com os valores obtidos por Lopes (2019) para os segmentos 04 e 05, tem-se a situação mostrada na Tabela 11.

O segmento 01 apresentou um ATR 45\% menor que o segmento 05 (espessuras de revestimento de $16,5 \mathrm{~cm}$ e $16,3 \mathrm{~cm}$, respectivamente). 


\section{Revisto Técrico-Cientifica de Engenharia Civil CIVILTEC}

Tabela 11: Comparativo entre as medidas de ATR dos segmentos com e sem MRAF

\begin{tabular}{cccccc}
\hline & \multicolumn{3}{c}{ com MRAF } & \multicolumn{2}{c}{ sem MRAF ${ }^{(1)}$} \\
Segmento & TRE $(\mathrm{mm})$ & Sd $(\mathrm{mm})$ & Segmento & TRE $(\mathrm{mm})$ & Sd $(\mathrm{mm})$ \\
01 & 4,69 & 1,40 & 05 & 8,67 & 2,97 \\
06 & 8,19 & 1,76 & 04 & 9,50 & 2,97 \\
\hline
\end{tabular}

Fonte: dados da pesquisa e Lopes (2019) ${ }^{(1)}$

O segmento 06 apresentou um valor de ATR 13\% menor que o segmento 04 (espessuras de revestimento de $15,0 \mathrm{~cm}$ e $15,2 \mathrm{~cm}$, respectivamente). Ressalta-se que a diferença entre os levantamentos é de 13 meses, ou seja, Lopes (2019) levantou os dados em novembro de 2018 e nesta pesquisa, os levantamentos de ATR foram realizados em dezembro de 2019. Assim, pode-se concluir que a diferença foi ainda maior, tendo em vista que os valores tendem a aumentar conforme mostrado no gráfico da Figura 3 elaborado com os dados de todos os levantamentos feitos por Luz (2017) e Lopes (2019)

Figura 3: Evolução dos afundamentos dos segmentos monitorados

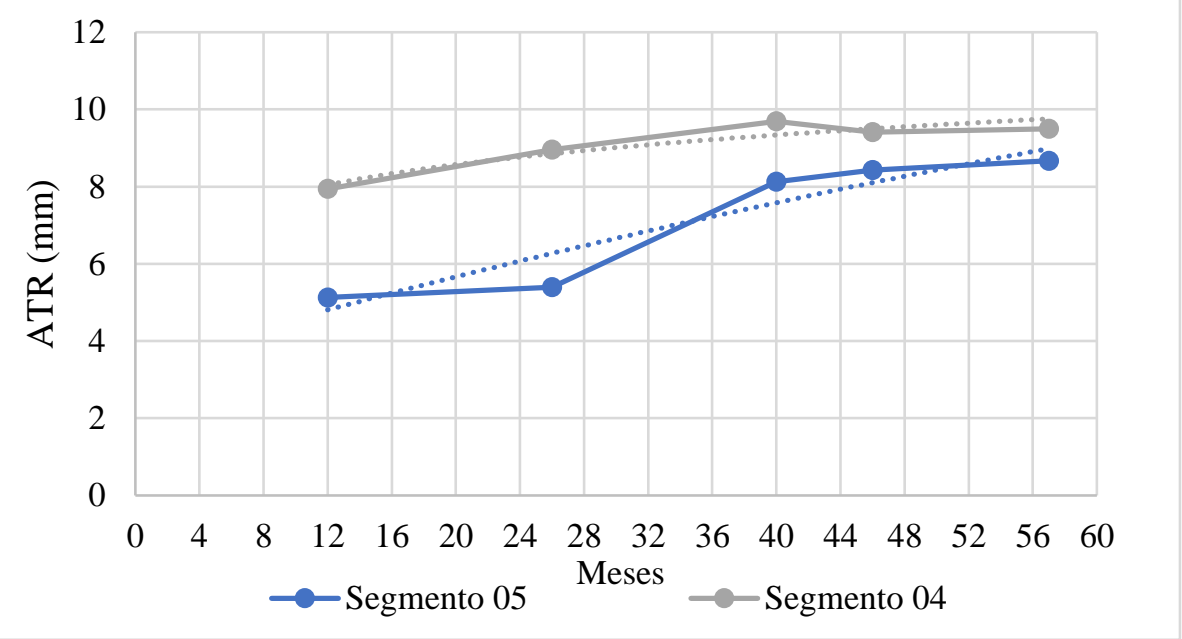

Fonte: Elaborado com dados de Luz (2017) e Lopes (2019).

Brasil (2006a) estabelece um limite de $12 \mathrm{~mm}$ de ATR máximo durante a vida útil dos pavimentos, acima disso, tem-se comprometimento estrutural e, sobretudo, prejuízo às condições de segurança da rodovia. Dessa forma, foi calculado através das equações obtidas por regressão, que ao final da vida útil definida em projeto (10 anos), os segmentos 04 e 05 teriam afundamentos em torno de 10,7 mm e 11,9 respectivamente, portanto muito próximo do limite estabelecido.

Como não há dados de monitoramento para os segmentos 01,02 e 06, não é possível prever o comportamento destes com relação ao afundamento na trilha de roda, no entanto, é 


\section{Revista Técnico-Cientifica de Engenharia Civil CIVILTEC}

possível perceber que o segmento 06 terá um comportamento semelhante aos segmentos 04 e 05, devido ao alto valor de ATR, conforme mostrado na tabela 8 , diferente do que ocorre nos segmentos 01 e 02 , já que apresentam valores bem satisfatórios.

A estimativa de vida útil dos segmentos 01,02 e 06 foi calculada utilizando o programa ViscoRoute. O software leva em consideração o comportamento viscoelástico das misturas asfálticas através dos parâmetros reológicos do modelo de Huet-Sayegh (tabela 3). Os parâmetros elásticos adotados nas simulações numéricas para as camadas de base, sub-base e subleito dos segmentos 01 e 02 foram os obtidos por Santos (2016) e Luz (2017). A Tabela 12 mostra os valores utilizados.

Tabela 12: Módulo resiliente (MR) e coeficiente de Poisson

\begin{tabular}{cccccc}
\hline \multicolumn{2}{c}{ Segmento 01 } & \multicolumn{2}{c}{ Segmento 02 } & \multicolumn{2}{c}{ Segmento 06 } \\
(1) \\
\hline MR (MPa) & Coef. Poisson & MR (MPa) & Coef. Poisson & MR (MPa) & Coef. Poisson \\
198 & 0,35 & 200 & 0,35 & 287 & 0,35 \\
188 & 0,35 & 191 & 0,35 & 228 & 0,35 \\
237 & 0,45 & 239 & 0,35 & 224 & 0,35 \\
\hline
\end{tabular}

Fonte: Santos (2016) ${ }^{(1)}$ e Luz (2017) ${ }^{(2)}$

A velocidade de tráfego e a temperatura do revestimento consideradas nessas simulações foram determinadas de acordo com os ensaios de fadiga. Quanto à velocidade de tráfego, assumiu-se $72 \mathrm{~km} / \mathrm{h}$ que corresponde à frequência de $10 \mathrm{~Hz}$. Em relação à temperatura, foi adotada a temperatura de $15^{\circ} \mathrm{C}$. Para o carregamento foi considerado o semieixo do eixo padrão rodoviário $(8,2 \mathrm{tf})$, com distância entre rodas de $32 \mathrm{~cm}$, carga por roda de $2050 \mathrm{kgf}$ e pressão de enchimento dos pneus de $5,6 \mathrm{kgf} / \mathrm{cm}^{2}$. A Tabela 13 apresenta os resultados da estimativa da vida útil dos segmentos a partir da simulação numérica.

Tabela 13: Estimativa de vida útil a fadiga dos segmentos

\begin{tabular}{cccc}
\hline Segmento & $\begin{array}{c}\text { Espessura do } \\
\text { Revestimento }\end{array}$ & $\varepsilon\left(\times 10^{-6}\right)$ & $\mathrm{N}_{8,2 \text { tf }}$ \\
\hline 01 & 16,5 & 55 & $5,43 \mathrm{E}+06$ \\
02 & 18,0 & 49 & $9,40 \mathrm{E}+06$ \\
06 & 15,0 & 58 & $4,22 \mathrm{E}+06$ \\
\hline Fonte: Elaborado
\end{tabular}

Fonte: Elaborado com dados de Trichês (2014)

A partir dos dados de monitoramento nos segmentos 04 e 05, Luz (2017) encontrou fatores de calibração entre as estimativas de vida útil determinadas com o ViscoRoute a partir dos ensaios de laboratório (fadiga com o aparato 4 pontos) e o trincamento levantado em campo 


\section{Revista Técnico-Cientifica de Engenharia Civil CIVILTEC}

considerando $10 \%$ de trincamento na área da trilha de roda. A Tabela 14 mostra os fatores de calibração encontrados pela autora para os segmentos 04 e 05 .

Tabela 14: Fator laboratório/campo para o aparecimento de $10 \%$ de trincamento na trilha de roda

\begin{tabular}{cccccc}
\hline $\begin{array}{c}\text { Previsão de início das } \\
\text { trincas }\end{array}$ & $\begin{array}{c}\text { Porcentagem } \\
\text { de } \\
\text { trincamento }\end{array}$ & $\mathrm{N}_{\text {lab }}$ & $\mathrm{N}_{\text {campo }}$ & $\begin{array}{c}\text { Fator } \\
\text { laboratório/campo } \\
f=\mathrm{N}_{\text {campo }} / \mathrm{N}_{\text {lab }}\end{array}$ & $\begin{array}{c}\text { Fator } \\
\text { adotado }\end{array}$ \\
\hline Segmento 04 & $10 \%$ & $3,90 \mathrm{E}+06$ & $1,05 \mathrm{E}+07$ & 2,69 & 3 \\
Segmento 05 & & $5,93 \mathrm{E}+06$ & $1,80 \mathrm{E}+07$ & 3,04 & 3 \\
\hline
\end{tabular}

Fonte: Adaptado de Luz (2017).

Com os resultados encontrados para os segmentos 04 e 05, é possível se fazer uma estimativa do que ocorreria nos segmentos da pista 01, 02 e 06 (pista sul), caso estes segmentos não tivessem recebidos a camada de microrrevestimento. $\mathrm{O}$ comparativo entre esses segmentos se justifica pois foram construídos simultaneamente (pista sul e pista norte), utilizando os mesmos materiais, variando-se apenas as espessuras do revestimento.

Aplicando os fatores de calibração nas estimativas de vida útil obtidas para os segmentos da pista sul, têm-se os resultados apresentados na Tabela 15.

Tabela 15: Estimativa de vida útil utilizando os fatores de calibração e a partir da Figura 4

\begin{tabular}{ccccccc}
\hline \multirow{2}{*}{ Segmento } & $\begin{array}{c}\text { Espessura } \\
\text { do Reves. }\end{array}$ & $\begin{array}{c}\mathrm{N}_{\text {usace }} \\
\text { (início do } \\
\text { (cm) }\end{array}$ & \multicolumn{2}{c}{$\mathrm{N}_{\text {usace }}$ (aparecimento de } & \multicolumn{2}{c}{$\mathrm{N}_{\text {usace }}$ (aparecimento de } \\
trincamento) & Simulação & Gráfico da & \multicolumn{2}{c}{ 20\%trincas) } \\
numérica & Figura 4 & numérica & Gráfico da \\
\hline 01 & 16,5 & $5,43 \mathrm{E}+06$ & $1,63 \mathrm{E}+07$ & $7,0 \mathrm{E}+07$ & $2,17 \mathrm{E}+07$ & $8,4 \mathrm{E}+07$ \\
02 & 18,0 & $9,40 \mathrm{E}+06$ & $2,82 \mathrm{E}+07$ & $8,6 \mathrm{E}+07 *$ & $3,76 \mathrm{E}+07$ & $9,8 \mathrm{E}+07 *$ \\
06 & 15,0 & $4,22 \mathrm{E}+06$ & $1,27 \mathrm{E}+07$ & $5,4 \mathrm{E}+07$ & $1,69 \mathrm{E}+07$ & $7,0 \mathrm{E}+07$ \\
\hline
\end{tabular}

Fonte: dados da pesquisa.

O acréscimo de desempenho proporcionado pela aplicação do microrrevestimento retardou o aparecimento das trincas na superfície e consequentemente gerou um aumento na vida útil do pavimento. Isto possibilitaria que a concepção do dimensionamento do revestimento em mistura asfáltica com ligante convencional com 18,0 cm de espessura e aplicação de 1,5 cm de microrrevestimento quando do aparecimento das primeiras trincas fosse suficiente para proporcionar o desempenho esperado do pavimento para atender ao tráfego de 8,02E+07. Em assim sendo, para os segmentos 01 e 06, se faz necessário adequar a espessura do revestimento para atingir a vida útil requerida em projeto. 


\section{Revista Técnico-Cientifica de Engenharia Civil CIVILTEC}

A partir dos fatores campo/laboratório mostrados na Tabela 14, Luz (2017) calculou as espessuras necessárias para que os segmentos 04 e 05 atendessem a vida útil definida em projeto considerando-se $10 \%$ de trincamento na trilha de roda. As espessuras encontradas foram, respectivamente, 21,5 e $20,5 \mathrm{~cm}$, ou seja, no segmento 04 precisaria ser colocado mais $6,5 \mathrm{~cm}$ de espessura para que no final da vida de projeto se atingisse cerca de $10 \%$ da área trincada e no segmento $05,4,0 \mathrm{~cm}$ de espessura.

Para um comparativo entre os custos da aplicação de microrrevestimento (solução empírica adota pelo DNIT para preservar a vida útil do pavimento) e proposição de aumento de espessura do revestimento (proposta por Luz, 2017, tendo em conta a análise mecanicista da estrutura), utilizou-se os dados do desempenho dos segmentos 02 e 05 onde o primeiro foi executado com $18 \mathrm{~cm}$ de revestimento asfáltico e o segundo, executado com $16,5 \mathrm{~cm}$ de revestimento asfáltico (ambos com mais 1,5 cm de microrrevestimento, aplicado 1 ano após a abertura ao tráfego).

A Tabela 16 mostra o custo da execução do microrrevestimento no segmento 02 na época da execução (março de 2015) e a Tabela 17 mostra os valores que seriam necessários para o aumento de espessura do revestimento do segmento 02, em março de 2014. Foi utilizado como referência o preço contratual na data de execução do microrrevestimento, ou seja, março de 2015, exatamente um ano após a abertura ao tráfego. Os valores também foram atualizados para janeiro de 2020.

Tabela 16: Custo da aplicação do microrrevestimento no segmento 02

\begin{tabular}{cccccc}
\hline $\begin{array}{c}\text { Área } \\
\left(\mathrm{m}^{2}\right)\end{array}$ & $\begin{array}{c}\text { Microrrevestimento } \\
\text { Preço Unitário } \\
\left(\mathrm{R} \$ / \mathrm{m}^{2}\right)\end{array}$ & $\begin{array}{c}\text { Valor } \\
(\mathrm{R} \$)\end{array}$ & $\begin{array}{c}\text { Transporte dos Agregados } \\
\text { Valor } \\
(\mathrm{R} \$)\end{array}$ & $\begin{array}{c}\text { Total }(\mathrm{R} \$) \\
\text { Março/2015 }\end{array}$ & $\begin{array}{c}\text { Total }(\mathrm{R} \$) \\
\text { Jan } / 2020\end{array}$ \\
\hline $2.100,00$ & 5,8 & $12.180,00$ & 366,23 & $12.546,23$ & $24.074,87$ \\
\hline
\end{tabular}

Fonte: dados da pesquisa

Tabela 17: Custo da solução de reforço de 4,0 cm

\begin{tabular}{|c|c|c|c|c|c|c|}
\hline \multicolumn{4}{|c|}{ Mistura Asfáltica Convencional $(4 \mathrm{~cm})$} & \multirow{2}{*}{$\begin{array}{c}\text { Transporte dos } \\
\text { Agregados } \\
\text { Valor } \\
(\mathrm{R} \$)\end{array}$} & \multirow{2}{*}{$\begin{array}{c}\text { Total }(\mathrm{R} \$) \\
\text { Março/2015 }\end{array}$} & \multirow{2}{*}{$\begin{array}{c}\text { Total }(\mathrm{R} \$) \\
\text { Jan/2020 }\end{array}$} \\
\hline $\begin{array}{l}\text { Área } \\
\left(\mathrm{m}^{2}\right)\end{array}$ & $\begin{array}{c}\text { Volume } \\
\left(\mathrm{m}^{3}\right)\end{array}$ & $\begin{array}{l}\text { Preço Unitário } \\
\left(\mathrm{R} \$ / \mathrm{m}^{3}\right)\end{array}$ & $\begin{array}{c}\text { Valor } \\
(\mathrm{R} \$)\end{array}$ & & & \\
\hline $2.100,00$ & 84,00 & 382,33 & $32.115,72$ & $2.907,29$ & $35.023,01$ & $67.761,05$ \\
\hline
\end{tabular}

Fonte: dados da pesquisa 


\section{Revista Técnico-Cientifica}

de Engenharia Civil

O acréscimo de custo da solução de reforço na camada de revestimento para resistir a vida útil definida $\mathrm{m}$ projeto é de 2,8 vezes para o critério de $20 \%(4 \mathrm{~cm})$ e de 3,5 vezes para o critério de $10 \%$ de trincamento na trilha de roda $(5 \mathrm{~cm})$.

É importante salientar que esses seriam os custos de se executar o reforço ainda ao final do primeiro ano de serviço, assim como ocorreu com a aplicação do MRAF. Na prática, o que ocorre na maioria dos contratos de manutenção rodoviária é a intervenção após o aparecimento de trincamento na superfície do pavimento, o que demanda maiores custos tendo em vista a necessidade de fresagem e recomposição da camada trincada.

\section{Conclusão}

A solução de aplicação de microrrevestimento asfáltico a frio - MRAF, inicialmente proposta como uma solução de auxílio na redução de propagação e agravamento de fissuras na superfície do pavimento mostrou-se assertiva e eficaz, proporcionando, até o momento, um aumento entre 52\% a 98\% na vida útil dos pavimentos. Essa melhoria no desempenho dos pavimentos também pode ser evidenciada nos valores de afundamento de trilha de rodas, que se apresentaram valores entre $13 \%$ e $45 \%$ menores que os obtidos nos segmentos que não receberam a camada de microrrevestimento.

Dessa forma, a técnica de utilização da camada de MRAF acarretou benefícios econômicos ao erário, tendo retardado a necessidade de intervenções no pavimento. Além disso, pode-se evidenciar que a espessura de $18 \mathrm{~cm}$ de revestimento aliada a camada de $1,5 \mathrm{~cm}$ de MRAF, pode vir a ser capaz de resistir a vida útil estimada em projeto, conforme demostrada pelas simulações numéricas através do programa ViscoRoute.

\section{Referências}

ABNT: Associação Brasileira de Normas Técnicas. ABNT NBR 14746: Microrrevestimentos a frio e lama asfáltica - Determinação de perda por abrasão úmida (WTAT). Rio de Janeiro. 2014.

ABNT NBR 14757: Microrrevestimentos e lamas asfálticas - Determinação da adesividade de misturas. Rio de Janeiro. 2017.

ABNT NBR 14841: Misturas asfálticas - Determinação da adesão de areia em microrrevestimentos asfálticos a frio por meio da máquina LWT. Rio de Janeiro. 2015. ABNT NBR 14841: Microrrevestimentos asfálticos - Determinação da coesão e características da cura pelo coesímetro. Rio de Janeiro. 2017. 
AFNOR - Association Française de Normalisation. AFNOR NF P 98-253-1: Préparation des mélanges hydrocarbonés, partie 1: essai d'orniérage. Association Française de Normalisation, AFNOR 1993.

BRASIL - Departamento Nacional de Infraestrutura de Transportes (DNIT). Manual de restauração de pavimentos asfálticos. Rio de Janeiro, 2006a.

CERATTI, J. A. P; REIS, R. M. M. Manual de Microrrevestimento Asfáltico a Frio MRAF. Instituto Pavimentar, 2011. 1998.

DNER ME 035: Agregados: determinação da abrasão "Los Angeles”. Rio de Janeiro,

DNER ME 054: Equivalente de areia. Rio de janeiro, 1997.

DNER ME 089: Agregados - determinação da durabilidade pelo emprego de soluções de sulfato de sódio ou de magnésio. Rio de Janeiro, 1994.

DNIT - Departamento Nacional de Infraestrutura de Transportes. DNIT 035/2005: Pavimentos flexíveis - Micro revestimento asfáltico a frio com emulsão modificada por polímero - Especificação de serviço. Rio de Janeiro. 2005.

EN - European Standard. EN 12697-24: Bituminous mixtures - test methods for hot mix asphalt, part 24: Resistance to fatigue. CEN, Brussels. 2004.

EN 12697-26: Bituminous mixtures - test methods for hot mix asphalt, part 26: Stiffness. CEN, Brussels. 2004.

ISSA - INTERNATIONAL SLURRY SURFACING ASSOCIATION. ISSA A143: Recommended perfomance guidelinefor micro-surfacing. USA. 2010.

LOPES, T. A. S. Análise do efeito da sazonalidade da temperatura e do tráfego no desempenho de pavimentos flexíveis: segmentos monitorados de Araranguá-SC. Dissertação (Mestrado) - Programa de Pós-Graduação em Engenharia Civil, Universidade Federal de Santa Catarina, Florianópolis, 2019.

LUZ, L. C. Desempenho de revestimentos asfálticos executados em camada singular e integradas em rodovias de tráfego pesado: segmentos monitorados de Araranguá. Dissertação (Mestrado) - Programa de Pós-Graduação em Engenharia Civil, Universidade Federal de Santa Catarina, Florianópolis, 2017.

MELO, J. V. S. Desenvolvimento e estudo do comportamento reológico e desempenho mecânico de concretos asfálticos modificados com nanocompósitos. Tese (Doutorado) Programa de Pós-Graduação em Engenharia Civil, Universidade Federal de Santa Catarina, Florianópolis, 2014.

SANTOS, A. S. Segmentos monitorados de Araranguá: Construção e previsão de desempenho do revestimento em camadas singulares e integradas. Dissertação (Mestrado) - Programa de Pós-Graduação em Engenharia Civil, Universidade Federal de Santa Catarina, Florianópolis, 2016.

TRICHÊS, G. Desenvolvimento de método para dimensionamento de pavimentos asfálticos. Fase I: trechos experimentais e materiais de pavimentação. Florianópolis, 2014 